\title{
Soziale Dienstleistungen in Kooperationen
}

\author{
Daseinsvorsorge durch Aufbau und \\ Steuerung interorganisatorischer Netzwerke
}

Michael Noack

Michael Noack M.A. ist staatlich examinierter Krankenpfleger und wissenschaftlicher Mitarbeiter am Forschungsschwerpunkt »sozial raum management « des Instituts für angewandtes Management und Organisation in der sozialen Arbeit an der Fachhochschule Köln. Seine Themenschwerpunkt ist sozialräumliche Organisations- und Netzwerkentwicklung. E-Mail michael.noack@fh-koeln.de
Die angemessene Erbringung von Leistungen sozialer Daseinsvorsorge liegt auch in der Verantwortung der Dienste und Einrichtungen Sozialer Arbeit. Die Kooperation in Netzwerken kann dazu einen gangbaren Weg bieten.

»Je spezialisierter und differenzierter die formalen Hilfesysteme werden, desto höher wird ihre Problemlösungskompetenz und desto geringer ihre Lebensweltorientierung. Die Folge davon ist allen bekannt: Die AdressatInnen irren von einer Stelle zur nächsten, ohne dass ihnen geholfen wird (...) «(Santen/Seckinger 2005: 204).

Dieses Zitat verdeutlicht eine der Schattenseiten ausdifferenzierter gesellschaftlicher Hilfesysteme: Die Entstehung von "Funktions- und Hierarchiebarrieren « (Schubert 2008: 20 ff.), die eine strukturell hochwertige Soziale Arbeit für die Adressatinnen und Adressaten erschweren. Eine Möglichkeit diesen Zustand ins Positive umzukehren, ist die Erforschung neuer Möglichkeiten der Erbringung sozialer Dienstleistungen.

Ziel eines Forschungsvorhabens in Nordrhein-Westfalen ist es, sowohl im Profit- als auch im Non-Profit-Bereich mögliche Unterstützungsbedarfe bei der zwischenbetrieblichen Zusammenarbeit $\mathrm{zu}$ explizieren. Dafür wurde in diesem Bundesland eine quantitative Vollerhebung u. a bei allen sechs Wohlfahrtsverbänden und den öffentlichen Trägern sozialer Dienstleistungen durchgeführt, an die vertiefende, qualitative Fallanalysen angeschlossen wurden. In Abgrenzung zu diesem Projekt habe ich die Daten im Rahmen meiner Master-Thesis genutzt, um mich mit Netzwerkkooperationen aus sozialwirtschaftlicher Perspektive zu beschäftigen. Dieser Perspektive lagen folgende Ziele zugrunde:

- Gewinnen von Erkenntnissen bezüglich der Entstehung von sozialem Kapital in Form immaterieller und materieller Effekte der Vernetzung
- Beleuchten von Möglichkeiten, immaterielle und materielle Effekte der Vernetzung für den Aufbau und die Steuerung interorganisatorischer Dienstleistungsangebote zu nutzen und

- Suche nach Konsequenzen für ein entsprechendes Netzwerkmanagement

Der vorliegende Beitrag fokussiert auf den immateriellen Vernetzungseffekt »Vertrauen«. Es werden Forschungsergebnisse dargestellt, wie der interorganisatorische Leistungsaustausch von vertraulichen Beziehungen zwischen den Organisationsvertretern profitieren kann. Auf dieser Grundlage erfolgt die Formulierung von Handlungsempfehlungen für die Vernetzung sozialer Organisationen auf der Grundlage vertraulicher Leistungsaustauschbeziehungen.

\section{Netzwerke und soziale Dienstleistungen}

Soziale Dienstleistungen sind "personenbezogene Dienstleistungen«, da sie »direkt an der Person « erbracht werden (Siegler 1997: 36). Hinsichtlich der Personenbezogenheit sozialer Dienstleistungen gilt es eine Unterscheidung zu treffen, nämlich die Differenzierung in primäre und sekundäre Dienstleistungsaktivitäten: »Die primären Aktivitäten beinhalten die Herstellung der Dienstleistung, ihre Distribution unter den AdressatInnen und den begleitenden Service. Die sekundären Aktivitäten sichern, dass die primären $\mathrm{Ak}$ tivitäten effizienter und effektiver stattfinden können. «(Schubert 2008: 23)

Für die Erbringung von sozialen Dienstleistungen ergibt sich dadurch die Notwendigkeit eines »integrierten Prozessdenkens « (Schubert 2008: 22). Damit ist gemeint, dass alle Aktivitäten, mit denen eine Dienstleitung entworfen, hergestellt, vertrieben und unterstützt wird, zu einer ganzheitlichen Kette von Prozessen verknüpft werden, was gegenwärtig nicht der Fall ist (vgl. Schubert 2008: 20 ff.). 


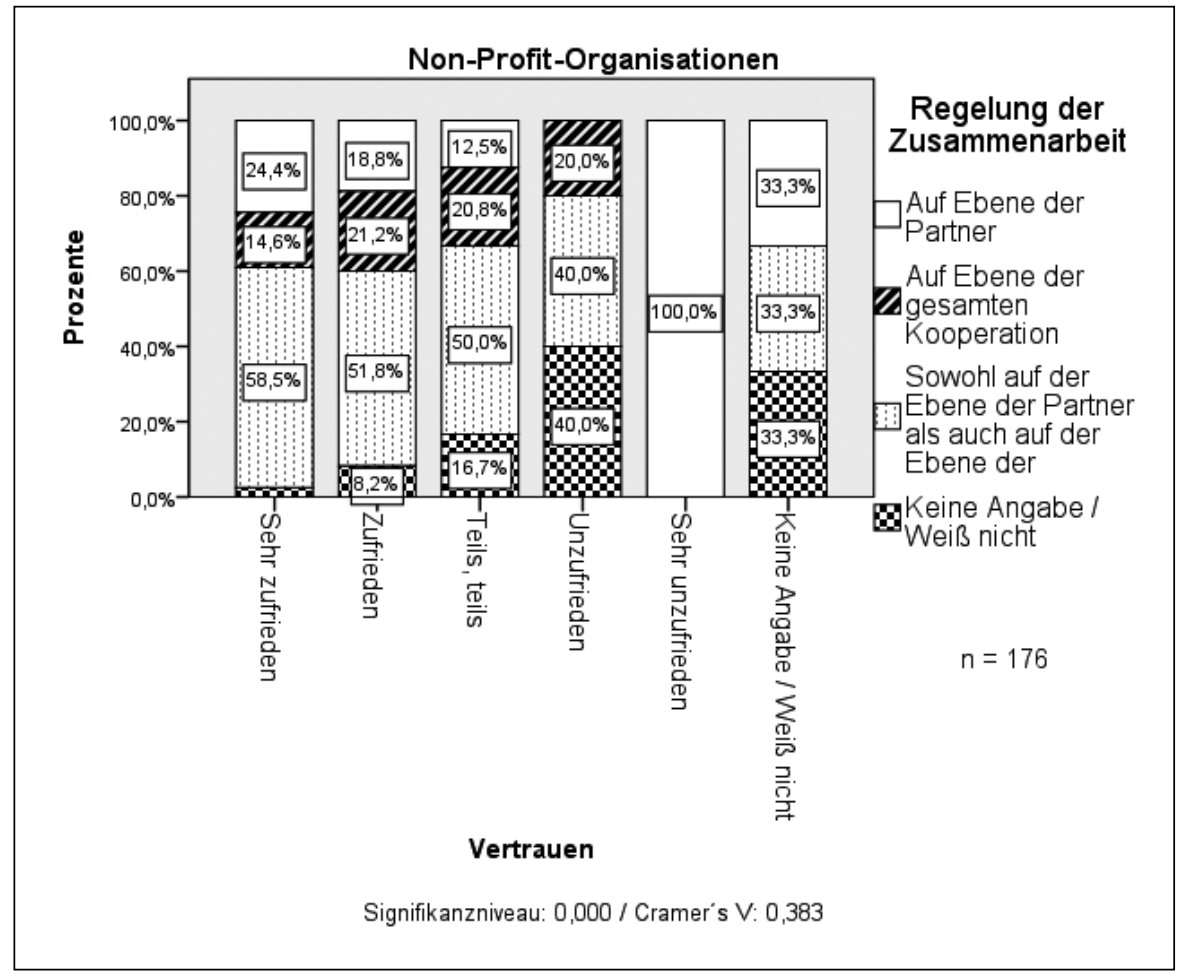

Abb. 1: Netzwerke, die sowohl auf zentralen wie auf dezentralen Regelungen basieren, rufen tendenziell am meisten Zufriedenheit hervor.

\section{Netzwerke und soziales Kapital}

In der Literatur wird der Begriff »soziales Kapital « bemüht, um den wirtschaftlichen Wert der Effekte sozialstrukturell eingebetteter Leistungsaustauschbeziehungen aufzuzeigen (vgl. Fuchs 2006: 77). Fuchs (ebd.: 105) definiert soziales Kapital als »eine Ressource, die in die soziale Struktur von Beziehungen eingebettet ist und Mitgliedern der begrenzten Menge von Kontakten in dem Netzwerk für ihre Handlungsinteressen zur Verfügung steht. Der Wert besteht darin, dass einzelne Mitglieder Interessen verwirklichen können, die sie ohne Zugriff und Nutzung auf die bereitgestellten Ressourcen im Netz von sozialen Beziehungen nicht nutzen können. «

Eine Ausprägung des sozialen Kapitals, das sich durch die Vernetzung ergeben kann und für wirtschaftliche Interaktionen und Transaktionen relevant ist, sind Vertrauensverhältnisse zwischen den Akteuren (vgl. Stolle/Rothstein 2007: 134).

Vertrauen als immaterieller Vernetzungseffekt entsteht auf der Mikroebene (relationaler Effekt) und übt sowohl auf der Mikro- als auch auf der Mesoebene (struktureller Effekt) einen wirtschaftlichen Effekt auf den zwischenbetrieblichen Leistungsaustausch (Transaktio-
Quelle: Eigene Berechnungen

gentlichen physischen Güteraustausch logisch, meist auch zeitlich vorausgeht. «

Die Hauptaussage dieser Denkschule besteht darin, dass jedes Problem des Leistungsaustausches ein Vertragsproblem ist (vgl. Williamson 1990: 20). Denn das Menschenbild, das dieser Theorie zugrunde liegt, geht davon aus, dass Leistungsaustauschpartner begrenzt rational sind und zu opportunistischen Verhalten neigen: »Diese zwei Verhaltensannahmen erlauben folgende Kurzfassung des Problems ökonomischer Organisation: Wie schafft man Vertrags-und Beherrschungsbzw. Überwachungssysteme, die der begrenzten Rationalität Rechnung tragen und zugleich Transaktionen gegenüber den Gefahren des opportunistischen Verhaltens absichern? « (ebd.: XI)

Opportunismus ist gemäß dieser Theorie als nicht » irrelevant « abzutun, dieses Verhalten erfordere zentrale Herrschaftsund Überwachungssysteme, »die im übrigen Vertrauen « (ebd. 72) schaffen sollen.

\section{Forschungsergebnisse}

nen) aus: »Der relationale Effekt resultiert aus der strukturellen Einbettung zwischen zwei Akteuren. Eine Tauschbeziehung ist eingebettet, sobald die Tauschpartner neben dem Preis- und Mengeninformationen, welche die Tauschleistung im engeren Sinn betreffen, weitere Informationen über den Gegenüber erlangen. Diese verdichten sich im Zuge wiederholter Transaktionen und führen dazu, dass Verhaltenserwartungen und Vertrauen zwischen den Tauschpartnern entstehen (...). Der strukturelle Effekt der Einbettung ergibt sich aus der Gesamtheit der Beziehungen und hat einen indirekten Einfluss auf das Handeln. Neben Kontroll- und Überwachungskosten werden auch Informations- und Koordinationskosten gesenkt.« (Vgl. Wald/Jansen 2006: 101)

\section{Netzwerke und Transaktionen}

Diesen theoretischen Annahmen stehen die Aussagen der Transaktionskostentheorie (vgl. Williamson 1992) gegenüber. Picot (1982: 269) definiert Transaktionen folgendermaßen: »Eine Transaktion umfasst den Prozess der Anbahnung, Vereinbarung, Kontrolle, und u. U. Anpassung eines Leistungsaustausches, der dem ei-
Durch eine Hypothese soll die Überlegung überprüft werden, wonach Herrschafts- und Überwachungssysteme Vertrauen erzeugen. Leistungsaustauschprozesse werden auf der Grundlage von Regelungen zur Zusammenarbeit beherrscht und überwacht. Daher wird folgende Hypothese geprüft: Unter dem Einfluss von Regelungen der Zusammenarbeit entsteht mehr Zufriedenheit mit dem Vertrauen. Es besteht ein Zusammenhang zwischen Regelungen der $\mathrm{Zu}$ sammenarbeit und der Zufriedenheit mit dem Vertrauen.

Befragte, die sehr zufrieden mit dem Vertrauen innerhalb ihrer Kooperation sind, haben:

- zu 24,4 Prozent angegeben, dass ihre Zusammenarbeit auf Ebene der Partner geregelt ist

- zu 14,6 Prozent in Netzwerken agieren, in denen die Zusammenarbeit auf der Kooperationsebene geregelt ist, und

- zu 58,5 Prozent angegeben haben, dass ihre Kooperation sowohl auf der Partner- als auch auf der Kooperationsebene geregelt ist (vgl. Abb. 1).

Die schwache Bestätigung dieser Hypothese zeigt an, dass eine Koordination, die auf zentralen und dezentralen Regelungen basiert, tendenziell am meisten $\mathrm{Zu}$ - 


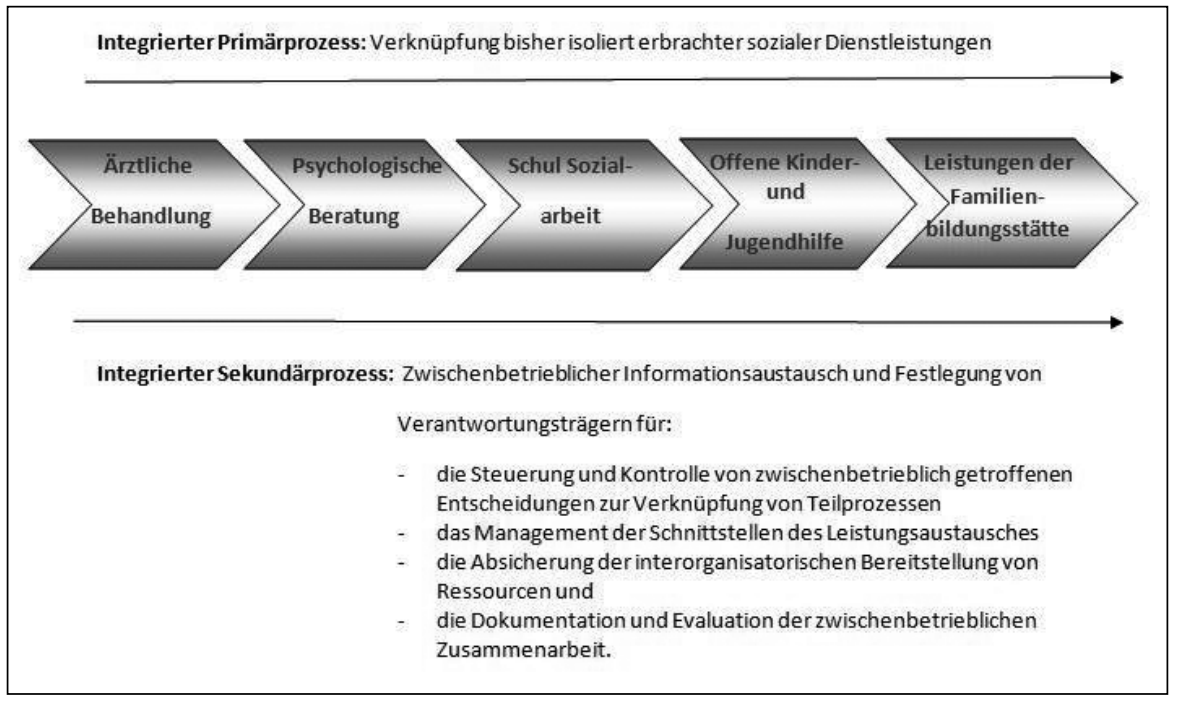

Abb. 2: Wenn Institutionen und Organisationen der Sozialen Arbeit vernetzt arbeiten, kann für hilfesuchende Menschen ein zusammenhängender Dienstleistungsprozess geleistet werden.

Quelle: In Anlehnung an: Gaitanides, M. (1983): Prozessorganisation Entwicklung, Ansätze und Programme prozessorientierter Organisationsgestaltung. Zitiert in: Schubert, H. (2008): Netzwerkkooperation - Organisation und Koordination von professionellen Vernetzungen. S.: 23.

friedenheit mit dem Vertrauen innerhalb einer Kooperation hervorruft.

\section{Handlungsempfehlungen}

Hinsichtlich der Genese von Vertrauen zwischen den Netzwerkpartnern durch die Koordination einer Kooperation wird zu Beginn der interorganisatorischen $\mathrm{Zu}$ sammenarbeit empfohlen, eine Win-winSituation für die Netzwerkpartner zielorientiert zu planen und vertraglich zu fixieren.

Dafür brauchen Netzwerkkooperationen Prioritätslisten für die angestrebten Ziele, in deren Mittelpunkt die Kostenträger, die Leistungsverbraucher (Kollektivziele) und die Netzwerkpartner (Individual- bzw. Teilziele) stehen (vgl. Bossong 2003: 474).

Es wird empfohlen, ein strategisches, kollektives Ziel für die Netzwerkkooperation $\mathrm{zu}$ formulieren und in einem Kooperationsvertrag zu verankern. Von diesem sind individuelle Teilzielsetzungen für die Netzwerkpartner abzuleiten, aus denen wiederum der Nutzen der $\mathrm{Zu}$ sammenarbeit für jeden einzelnen Organisationsvertreter hergeleitet werden kann.

Die Ziele sollten dabei nicht nur exakt formuliert werden, sondern nach dem SMART-Prinzip auch spezifisch, messbar, attraktiv, realistisch und terminfi- der Netzwerkpartner zunächst auf der Grundlage eines Vertrages auf der Kooperationsebene planen und überwachen. In diesem Vertrag sind Kostenfragen bezüglich der gemeinsamen Dienstleistungserbringung und Verknüpfungsmodi von Teilleistungen und der Anpassungen von Fehlentwicklungen zu regeln.

Trotzdem sollte ein solcher Kontrakt genug Freiraum für spontane Interaktionen zwischen den Organisationsvertretern auf der Mikroebene lassen. Dann können die Netzwerkakteure ihren Ideenreichtum nutzen und so schrittweise eine gemeinsame Handlungsverantwortung im interorganisatorischen Netzwerk herausbilden. Zudem kann sich Vertrauen im Netzwerk reproduzieren, wenn die Netzwerkkoordination den Netzwerkpartnern Autonomie vermittelt. Insofern wird eine Mischung aus strategischen Koordinationselementen (Allopoiese) auf der Kooperationsebene und der Mesoebene des Netzwerks und der Ermöglichung von Selbststeuerungsmechanismen hinsichtlich Zusammenarbeit zwischen einzelnen Netzwerkpartnern (Autopoiese) auf der Mikroebene empfohlen.

Durch vertrauliche Netzwerkbeziehungen können sich, innerhalb der formalen Rahmung durch einen Vertrag auf der Kooperationsebene informelle oder formelle Strukturen zur Fehlerdiagnose und Fehlerbehebung auf der Partnerebene herausbilden. Zur Unterstützung der sich entwickelnden Koordination des Leistungs- und Informationsaustausches auf einer Vertrauensgrundlage durch die Netzwerkpartner werden die Protokollierung der diesbezüglichen Absprachen und die netzwerkinterne Verbreitung der Protokolle empfohlen.

Es wird dringend angeraten, Netzwerke auf der Kooperationsebene in einem vertraglichen Rahmen zu fassen. Denn im Zuge vertiefender qualitativer Fallanalysen wurde festgestellt, dass immer dann Unterstützungsbedarf bei der Koordination der Zusammenarbeit angegeben wurde, wenn kein Kooperationsvertrag zwischen allen Netzwerkpartner geschlossen wurde.

\section{Fallbeispiel Janne}

Mit einem Fallbeispiel können die Ausführungen zu den theoretischen Grundlagen in Verbindung mit dem Forschungs- 
ergebnis und den daraus resultierenden Handlungsempfehlungen illustriert werden.

Janne ist elf Jahre alt. Er besucht die 5. Klasse einer Realschule. Dort fiel der Klassenlehrerin auf, dass er:

- die Regeln der Klassengemeinschaft nicht akzeptiert, den Unterricht wegen seiner geringen Ausdauer und seinem chaotischen Verhalten stört

- sich von seinen Mitschülern schnell ablenken lässt und

- eine geringe Frustrationstoleranz aufzeigt, was zu Wutanfälle und aggressivem Verhalten führt

Nachdem die Klassenlehrerin Rücksprache mit Jannes Eltern gehalten hat, welche diese Verhaltensweisen auch im Familienalltag feststellen, suchen diese den Kinderarzt auf, welcher bei Janne eine Aufmerksamkeitsdefizit-Hyperaktivitätsstörung (ADHS) diagnostiziert und die medikamentöse Behandlung mit Ritalin empfiehlt.

Da Jannes Eltern die Chancen und Risiken dieses Medikaments nicht kennen, wollen sie sich beraten lassen. Der Kinderarzt empfiehlt die örtliche psychologische Beratungsstelle, mit der seine Praxis vernetzt ist.

Der Leiter der psychologischen Beratungsstelle, Herr M., hat die Zusammenarbeit mit dem Kinderarztpraxis und weiteren Institutionen, die soziale Dienstleistungen für Menschen mit einer ADHS-Diagnose erbringen, initiiert. Dazu gehören die Schulsozialarbeiter in den städtischen Schulen, die Sozialarbeiter in Einrichtungen der offenen Kinderund Jugendarbeit sowie die örtliche $\mathrm{Fa}$ milienbildungsstätte. Es sind Akteure, mit denen Herr M. schon über Jahre hinweg immer wieder bei entsprechenden Fallindikationen zusammenarbeitete. Er veranlasste, dass sich die Akteure vierteljährlich zusammenfinden, um strategische Ziele für ihre Zusammenarbeit zu besprechen und vertraglich zu fixieren. Aktuell besteht die Strategie darin, passgenaue Hilfen zur richtigen Zeit am richtigen Ort zu ermöglichen. Die Zusammenarbeit funktioniert gut, weil die Netzwerkpartner durch ihren Kooperationsvertrag einen Orientierungsrahmen geschaffen haben, von dem sie operative Ziele für ihre konkret stattfindende Zusammenarbeit ableiten können. Zwischen den Partnern hat sich dadurch Vertrauen dahingehend entwickelt, dass ihre gegenseitigen Erwar- tungen und Verpflichtungen beispielsweise hinsichtlich der Weitergabe von datenschutzrechtlich unbedenklichen Informationen über gemeinsame Fälle erfüllt werden. Aufgrund dieser vertraulichen Leistungsaustauschbeziehungen halten sie sich an Absprachen, die sie im Zuge der fallweisen Zusammenarbeit getroffen haben.

Herr M. erklärt Janne und seinen Eltern, dass bei der Diagnosestellung die sozialen Komponenten wie zum Beispiel die Familie und die neurologischen Komponenten - hierzu zählt der Gehirnstoffwechsel - getrennt voneinander betrachtet werden. Darin liegt die Schwierigkeit der Behandlung. Denn die sozialen und neurologischen Komponenten beeinflussen sich gegenseitig. Deswegen hat sich Herr M. mit den beschriebenen Institutionen und ihren Vertretern vernetzt, was dazu führt, dass datenschutzrechtlich unbedenkliche Informationen über gemeinsame Fälle zwischen den Netzwerkpartner ausgetauscht und ihre Dienstleistungen, orientiert an den Bedürfnissen von Janne und seiner Familie aufeinander abgestimmt werden können. Daher kann er mit der Familie einen Behandlungsplan erstellen, in den sowohl die Dienstleistungen der Kinderarztpraxis, als Verantwortungsträger für die neurologische Kom- ponente, als auch die Dienstleistungen der Schulsozialarbeit, der psychologischen Beratungsstelle, des Jugendzentrums und der Familienbildungsstätte, als Verantwortungsträger für die soziale Komponente,mit einbezogen werden.

Jannes Eltern mussten nicht von einer Stelle zur nächsten irren, ohne dass ihnen geholfen wurde. Denn die für den »Fall Janne « zuständigen Institutionen und Organisationen waren vernetzt und konnten ihre Teildienstleistungen orientiert an Jannes Bedürfnissen zu einem zusammenhängenden Dienstleistungsprozess integrieren (vgl. Abb. 2).

\section{Resümee}

Durch ein interorganisatorisches Netzwerk kommt man dem Grundanliegen flexibler sozialer Dienstleistungen näher: Die Spezialisierung der Hilfen zugunsten flexibler Reaktionsmöglichkeiten auf Anforderung aufzugeben und gleichzeitig Kooperationsmöglichkeiten in benachbarte Handlungsfelder zu schaffen. Diese Reaktionsmöglichkeiten stellen keine Ergänzung bestehender Hilfen dar, sondern sind eine Alternative zu zuversäulten Hilfesystemen (vgl. Rüth/Ruppel 2005: 95).

\section{Literatur-und Quellenangaben}

Fuchs, M.(2006): Sozialkapital, Vertrauen und Wissenstransfer in Unternehmen. Wiesbaden: Deutscher Universitätsverlag.

Picot, A.(1982): Transaktionskostenansatz in der Organisationstheorie: Stand der Diskussion und Aussagewert. In: DBW, 42 (2), S.: 267-284.

Rüth, C.; Ruppel, M.(2005): Arbeiten im und am »defizitären Feld «. Stadtteilbezogene Flexible erzieherische Hilfen und Kriseninterventionen. In: Kalter, B.; Schrapper, C. (2005) (Hg.): Was leistet Sozialraumorientierung? Konzepte und Effekte wirksamer Kinder-und Jugendhilfe. Weinheim: Juventa. S.: 95-101.

Schubert, H.(2008): Netzwerkkooperation - Organisation und Koordination von professionellen Vernetzungen. In: Schubert, H. (Hg.) (2008): Netzwerkmanagement. Opladen: Leske und Budrich. S.: 7-106.

Siegler, B., F.(1997): Ökonomik Sozialer Arbeit. Freiburg im Breisgau: Lambertus. Stolle, D.; Rothstein, B.(2007): Institutionelle Grundlagen des Sozialkapitals. In: Franzen, A.;Freitag, M. (2007); Sozialkapital. Grundlagen und Anwendungen. Köln: Kölner Zeitschrift für Soziologie und Sozialpsychologie. Sonderheft 47/2007.

Wald, A.; Jansen, D.(2006): Netzwerke. In: Benz, A.; Lütz, S.; Schimank, U.; Simonis, G. (2006):Handbuch Governance. Theoretische Grundlagen und empirische Anwendungsfelder. S.: 93-106.

Williamson,O., E.(1990): Die ökonomischen Institutionen des Kapitalismus. Tübingen: Mohr. 